UDK 92 Reicha A. J.

\title{
ANTOINE JOSEPH REICHA, AMI DE JEUNESSE DE L. VAN BEETHOVEN
}

\author{
Jiří Vysloužil (Brno)
}

La personnalité et l'oeuvre d'A. J. Reicha (né le 26 février 1770 à Prague, mort le 28 mai 1836 à Paris), musicien d'origine tchèque, émigré à Paris, ne peuvent être mieux caractérisées typologiquement que sous l'aspect que nous avons évoqué dans notre titre. En effet, A. J. Reicha, neveu et élève du célèbre compositeur tchèque Joseph Reicha (1752-1795), violoncelliste et directeur d'un opéra de cour, a vraiment mérité ce titre d'ami, car il a été dans les années 1785-1792 le compagnon de Beethoven. Tous les deux sont nés la même année (1770) et ils ont commencé leur carrière dans la même ville (Bonn), comme élèves des mêmes maîtres de musique (Joseph Reicha, pour A. J. Reicha il n'est pas certain qu'il ait été l'élève de Ch. $\mathrm{H}$. Neefe); ils ont ensuite été membres de l'orchestre et du choeur de la chapelle de l'Electeur et de l'orchestre du théâtre de Bonn et ils ont été tous les deux étudiants à l'Université de Bonn qui venait d'être fondée. ${ }^{1}$ Les données concernant ces premières années d'études et d'activités communes nous sont fournies par l'autobiographie de A. J. Reicha (ed. J. Vysloužil sous le titre Notes sur Antoine Reicha, Brno 1970), où en parlant de ses études Reicha ne nomme pas directement Beethoven, mais rappelle cette amitié par la phrase suivante: "Les élèves qui y (c'est-à-dire à l'Université de Bonn, note J. V.) firent des progrès marquants furent mes amisu. ${ }^{2}$

Une amitié d'études et de carrière artistique durant plusieurs années représente sûrement une phase importante pour la vie des deux artistes surtout pour A. J. Reicha. Le seul fait qu'A. J. Reicha ait fait partie des amis de Beethoven a rehaussé la biographie d'A. J. Reicha et l'a classé dans la galerie des compositeurs connus de la fin du 18ème et du début du 19ème siècle. Les années communes que Beethoven et Reicha passè-

1 A. J. Reicha a été immatriculé à l'Université de Bonn en même temps que L. van Beethoven et $K$. Kügel le 14 Mai 1789 et il suivait avec eux les cours sur la philosophie de Kant, sur la poésie lyrique, la métaphysique, sur la littérature et l'éthique grecques, comparer L. Schiedermair, Der junge Beethoven, Leipzig 1925, p. 195-196. A. Reicha était lié d'amitié avec L. van Beethoven de 1785 jusqu'à la fin d'octobre 1792 , en 1802 ils se sont de nouveau rencontrés à Vienne.

2 L'oeuvre citée page 16. Notes sur Antoine Reicha citent le nom de Beethoven jusqu'a la page 18. 
rent à Bonn ont eu leur intérêt plus tard dans l'historiographie musicale. A. W. Thayer, Ernst Bücken et Ludwig Schiedermair qui ont consacré une monographie aux années de jeunesse de Beethoven ${ }^{3}$ ont les mêmes vues sur A. J. Reicha (Schiedermair en plus avec Joseph Reicha). Ce sont eux qui sont les auteurs des premières études monographiques rédigées scientifiquement. Ils y ont surtout mis l'accent sur les faits biographiques, sur l'apport théorique d'A. J. Reicha et ont jugé beaucoup plus sobrement l'étendue de son activité de compositeur. Schiedermair et surtout ensuite Bücken ont indirectement contribué à créer une fausse idée sur A. J. Reicha comme étant d'abord un théoricien et en second lieu un compositeur. Dans le Riemann Musik-Lexikon (Personenteil L-Z, 1961, p. 479) par exemple, A. J. Reicha est classé comme: "französischer Musiktheoretiker und Komponist tschechischer Geburtı ${ }^{4}$, jugement semblabe à celui de J. F. Fétis (ibid. Personenteil A-K, 1959, p. 505) qui le nomme "belgischer Musikforscher und Komponistu.

Le fait que le nom d'A. J. Reicha s'est trouvé lié avec celui de Beethoven a sans doute avantagé Reicha d'une part, mais d'autre part, face à ce titan musical que fut Beethoven, A. J. Reicha s'est trouvé dans la position peu enviable d'un compositeur satellite d'un grand maître. Les jugements qui classent A. J. Reicha, auteur de plusieurs centaines d'oeuvres de tous genres et formes, ${ }^{5}$ seulement ou surtout comme un théoricien de la musique ou qui minimisent les facteurs les plus progressistes de son apport de créateur et de compositeur ne font, à notre avis, que repousser injustement son importance de compositeur à la périphérie de l'évolution de la musique classique et des débuts du romantisme.

Cette fausse idée a pu provenir de la comparaison avec Beethoven qui, lui, n'a écrit aucune oeuvre théorique, alors que "la liste des oeuvres" de A. J. Reicha comporte à côté des oeuvres musicales, des traités theoriques importants comme: Art du compositeur dramatique au cours complet de composition vocale, divisé en quatre parties et accompagné d'un volume de planches (1833, traduction allemande 1835), Traité de haute composition, faisant suite au Cours d'harmonie pratique et au Traité de mélodie (1824, 1826, traduction allemande 1834), Ueber das neue Fugensystem, als Zusatz zu 36 Fugues composées d'après un nouveau

3 L'oeuvre citée de Thayer, Schiedermair et celle de E. Bücken Beethoven und Anton Reicha, in: Die Musik XII - 1913, 2. Märzheft, p. $431-435$ et Anton Reicha als Theoretiker, in: Zeitschrift für Musikwissenschaft II-1919/20, p. 156 -170 .

4 Une attention suffisante n'est pas consacrée à l'oeuvre de composition d'A. J. Reicha dans la lere monographie complète de Maurice Emmanuel, Antonin Reicha, biographie critique, Paris 1937, et Olga Šotolová, Antonin Rejcha, pedagog a skladatel - »a. Rejcha pédagogue et compositeur», in: Hudební věda 1970, WII. récemment Stephan Kunze doute même de l'apport d'A. J. Reicha dans la composition de la fugue. Comparer Anton Reichas "Entwurf einer phrasierter Fuge», Zum Kompositionsbegriff im frühen 19. Jahrhundert, in: Archiv für Musikwissenschaft XV-1968, p. 289-307.

5 Pour l'étendue de l'oeuvre d'A. J. Reicha comparer ma Liste dans l'édition citée: Notes sur Antorne Reicha, p. 73-85, dans laquelle nous trouvons par exemple 9 opéras, 12 symphonies, environ 20 sonates, 11 ouvertures, 25 quintettes pour instruments à vent, environ 84 fugues, 1 Requiem, 1 Te Deum et d'autres. 
système pour Clavecin (1805) aj.6 Le classement d'A. J. Reicha comme théoricien de musique a pourtant un fondement rationnel: accentuer son apport évident dans la théorie musicale. (A. J. Reicha n'a vraiment pas de partenaires qui puissent lui être comparés parmi ses contemporains). J. F. Fétis a, il est vrai, dans son oeuvre Biographie universelle des musiciens ( 2 1864-VII, p. 180-181), rappelé la dépendance d'A. $\mathrm{J}$. Reicha des théoriciens de musique du $18^{\mathrm{e}}$ siècle (F. W. Marpurg, J. Ph. Kirnberger et peut être aussi J. Mattheson). Mais A. J. Reicha n'a pas été seulement un éclectique. Son système théorique comporte plusieurs idées. et éléments nouveaux et progressistes. Au moment de l'influence classique dominante de la diatonique majeure mineure dans la théorie de la mélodie et de l'harmonie, A. J. Reicha a apporté une conception dynamique dans laquelle il a introduit d'autres genres de gammes à côté des diatoniques et chromatiques, à côté des majeures et mineures et même modales (qu'on appelle liturgiques) et même à côté des gammes à demi-tons qui devaient comporter des différences d'un quart de ton des demi-tons. A. J. Reicha introduit aussi de nouveaux éléments dynamiques dans la théorie des formes et dans la phraséologie, et ainsi ouvre à la phrase musicale classique un espace dans l'utilisation des mètres musicaux impairs composés.?

Les traits novateurs du système théorique musical d'A. J, Reicha ont des traits spécifiques qui le classent parmi les personnalités modernes dynamiques du genre d'A. Schönberg, L. Janáček, A. Hába. En effet, dans sa théorie musicale, A. J. Reicha généralise les principes de composition de son oeuvre. Il explique dans sa théorie sur la mélodie, l'harmonie, les formes (surtout pour la fugue), dans la métrique et la rythmique ce qu'il a auparavant découvert, reconnu vrai. Même si A. J. Reicha n'avait pas écrit d'oeuvre théorique, l'historiographe de la musique devrait le reconnaître comme un compositeur créateur de nouvelles formes.

Que fut donc alors A. J. Reicha comme compositeur et quelles sont les valeurs dynamiques qui se trouvent dans son oeuvre si vaste?

A. J، Reicha a vécu et créé à la limite de deux époques de styles: le classicisme et le romantisme. Ses liens avec la première époque sont caractérisés par l'apport de la personnalité et de l'oeuvre de Joseph Haydn. Il a connu personnellement ce maître ainsi que ses oeuvres déjà lors de son séjour à Bonn (1787-1794) et à Hambourg (1794-1799). Pour A. J. Reicha, J. Haydn a sûrement représenté une des personnalités dominantes de l"histoire de la musique, le plus grand compositeur contemporain. $\mathrm{Ce}$ fut peut-être justement Haydn qui a attiré A. J. Reicha à Vienne après un séjour de deux ans à Paris $(1800-1802)$. Ce sont ses sentiments réels d'admiration qui expliquent le fait qu'il est devenu son élève à l'âge de 32 ans. Et il ne fut certainement pas un élève ordinaire. Avant de devenir

\footnotetext{
6. Le traité sur la fugue est la première oeuvre théorique éditée séparément, dans le manuscrit plusieurs traités théorique sosnt restés inédits. Le traité sur la mélodie a été édité séparément dejà en 1814, le traité sur l'harmonie en 1818. Comparer Notes sur Antoine Reicha, p. 83-85.

7 Dans la théorie de la musique, un nouveau sujet d'étude d'A. J. Reicha fut celui de la chanson populaire.
} 
élève de J. Haydn, il avait déja édité deux symphonies (opus 41 et 42 ), une ouverture en do majeur opus 24, il avait composé deux opéras et de nom. breuses oeuvres pour piano et autres oeuvres instrumentales (entre autres des sonates, 12 fugues pour piano, qu'il a plus tard ajoutées au volume de 36 fugues pour piano) etc. Ses études chez J. Haydn avaient plutôt, d'après ses propres paroles, le caractère de discussions sur l'art et la musique? Il avait fait ses études proprement dites bien avant de connaître J. Haydn, et avait appris, probablement comme autodidacte, toutes les finesses stylistiques et de composition du style classique tel qu'il est représenté dans les symphonies de pleine maturité de J. Haydn. Les deux symphonies d'A. J. Reicha opus 41 et 42 dont nous venons de parler, composées dans les années $1799-1800$, sont, ainsi que la I. symphonie en do majeur opus 21 (1799) de L. v. Beethoven, des oeuvres qui rappellent leur modèle non seulement par des traits et des divisions de forme (partie allegro avec introduction lente, partie lente, menuet, finale), mais aussi par l'esprit de leur musique. Les op. et 42. d'A. J. Reicha ne sont pas, par leur contenu, au dessous du style classique à, son apogée, et classent leur auteur parmi les élèves les plus marquants de J. Haydn, avec le jeune Beethoven.

La période "classique» d'A. J. Reichá est liée à son séjour à Vienne (1802-1808). Dans cette période il a écrit plus de 50 pièces de forme et de style classiques, entre autres 4 sonates pour piano op. $43,(3), 4$ sonates pour violon et piano op. 44, 55 (2), 62, 8 quatuors pour instruments à cordes op. 48 (3), 49 (3), 52, 58 et nombre d'autres pièces pour ensemble de chambre qui ont été éditées successivement, sur la recommandation de L. v. Beethoven ${ }^{9}$ chez l'éditeur de Leipzig Breitkopf \& Härtel et A. Kühnel. Aujourd'hui nous ne connaissons la plupart de ces pièces que par les vieilles éditions, des index d'éditions ou des catalogues récents. Il est resté sous la forme manuscrite 8 quintettes pour instruments à cordes, que le compositeur estimait au-dessus de tout à cause de leur ioriginalité et des exigences techniques». (Notes, p. 28). Dans les années 1808-1809, apparurent quatre nouvelles symphonies (plus tard complétées par six autres). Ces pièces aussi sont restées dans les archives, la plupart dans la Bibliothèque de Paris où elles attendent de reparaître au jour.

Pour l'historiographe de la musique, cette riche moisson des années 1802 à 1818 représente une preuve indiscutable de son appartenance à "l'école classique" viennoise qui a atteint le sommet de sa renommée avec Haydn, Mozart et Beethoven. Mais celle-ci a été préparée par des générations de compositeurs tchèques émigrés à Mannheim (J. V. Stamic), en Souabe à Marbourg Oettingen-Wallerstein et à Bonn (J. Reicha), à Berlin et ailleurs en Allemagne (František et Jiři Benda) etc. ${ }^{10}$ A. J. Reicha s'est

8 Comp. Notes sur Antoine Reicha, p. 24.

9 La lettre de L. van Beethoven à l'éditeur Härtl (22. 1. 1803) dans laquelle il recommande d'éditer les oeuvres d'A. J. Reicha qui sont wauch recht schön.... und ... wrecht fleißig gearbeitet«. L'oeuvre citée de Thayer, II. p. 616.

10 A cause de cela nous ne comprenons pas A. J. Reicha comme un compositeur émigré de la branche parisienne (française) comme nous le lisons chez Vladimir Helfert Česká moderní hudba - "La musique tchèque moderne», 
réclamé de cette école même après s'être définitivement installé à Parị et après avoir fusionné avec le milieu culturel français."1 L'ensemble des 25 quintettes pour instruments à vent, composés de 1811 à 1820 et publiés chez l'éditeur Janet et Co à Paris, en quatre fascicules op. 88, 91, 100 (le premier en date de tous les quintettes en fa de 1811 est resté manuscrit et se trouve à Bibliothèque Universitaire de Prague) représentent par leur contenu les valeurs dominantes du "classicisme" d'A. J. Reicha. D'ailleurs, dans ces quintettes pour instruments à vent, il ne dépasse pas l'horizon du classicisme musical de Haydn et de Mozart. Ces oeuvres gardent pour fond le modèle de la sonate-symphonie, plus précisément de la sonatequatuor de Haydn. Néanmoins nous pouvons indiquer comme une certaine nouveauté de ces oeuvres la modification du modèle classique dans les pièces pour ensemble de chambre à cinq voix et techniquement l'accord idéal des instruments à vent que la littérature musicale ne connaissait pas avant A. J. Reicha.12

A. J. Reicha n'a donc pas été seulement un satellite éclectique de "l'école classique" viennoise. Il a contribué à sa diversité de genres et à la formation de son style par de nombreuses oeuvres, parmi lesquelles les quintettes pour instruments à vent lui ont assuré une place importante dans l'histoire de la musique. Ces oeuvres seules suffiraient à le classer comme un compositeur remarquable. Il est avec Jan Ladislav Dusík (1760 -1812) une des personnalités éminentes de la troisième et dernière vague de l'émigration tchèque musicale, ${ }^{13}$ contemporaine de l'école de $\mathrm{V}$. J. Tomášek (J. H. Vořišek, F. M. Kníže) et des compositeurs de la Věnec Zbern Vlastenských - "Couronne des chants patriotiques" (František Skroup) qui ont jeté les bases de la musique nationale tchèque.

Les bases du style classique et les formes musicales de l'oeuvre d' A. J. Reicha n'épuisent pas le contenu et le dynamisme de sa composition.

Olomouc 1936, p. 172 et après lui surtout chez Jan Racek, Ceská hudba - "La musique tchèque», Praha 1958, p. 60, mais comme un des représentants caractéristiques de l'époque classique viennoise.

11 Entre autres comme un compositeur d'opéras sur des textes français, comme un auteur de traités théoriques écrits dans un français parfait, aussi bien que comme professeur au Conservatoire de Paris. Pour cela comparer Jirí Vysloužil, Antonín Rejcha und die tschechische Musik, in: SPFFBU H7, Series musicologica Brno 1972, p. 53-62. Pour Fétis A. J. Reicha n'a jamais cessé d'être un représentant de l'école allemande. Derrière cette conception nous devons chercher un phénomène musical dont la substance est formée par des caractères et des particularités du nom et de notion (Germania) c'est-à-dire l'Allemagne - le pays de tendances culturelles et territoriales non nationales, comme sait l'expliquer l'historiographie de la musique allemande par exemple dernièrement Heinrich Besseler (comparer son entête Deutsche Musik, in: Riemann, MusikLexikon, Sachteil, p. 218).

12 Pour cela comparer Alena Krutová: Les quintettes pour instruments à vent d'Antoine Reicha, Colloquium Musica cameralis, Brno 1971, sous presse, et Jirí Kratochvíl: Několik poznámek k historii dechového kvintetu, "Quelques notes sur l'histoire du quintette à vents», in: Hudební věda 1970, VII.

$13 \mathrm{La}$ première vague d'émigration était composée de maîtres baroques autour de Jan Dysmas Zelenka (1679-1745), la deuxième vague par les compositeurs préclassiques en tête avec Jan Václav Stamic (1717-1757), Jiř́ Benda (1722-95) et son frère František (1709-86), Josef Mysliveček (1737-81) et la troisième vague par la génération A. J. Reicha et J. L. Dusík. 
Cette partie de l'oeuvre d'A. J. Reicha a néanmoins contribué à l'incorporer au courant progressiste qui, dans l'Europe centrale, a brisé la suprématie de la musique religieuse de style baroque (parmi les nombreuses oeuvres d'A. J. Reicha nous pouvons compter au plus deux oeuvres religieuses: Missa pro defunctis-Requiem et Te Deum) ${ }^{14}$ Ce caractère presque unique. ment "profane" de l'oeuvre d'A. J. Reicha, à son époque presque un cas unique, ne peut être explique que difficilement et seulement musicalement, c'est-à-dire par son éducation musicale et son orientation artistique antérieures vers les formes musicales absolues du classicisme. La personnalité dynamique d'A. J. Reicha sur laquelle nous avons eu la possibilité d'attirer l'attention lors d'une brève caractéristique de son oeuvre théorique était évidemment déterminée d'une part par son caractère et d'autre part par quelques événements de sa vie, qui à la fin l'ont placé dans la situation de pionnier de la poétique musicale (de la conception artistique) du XIX $\mathrm{X}^{\mathrm{e}}$ siècle.

Rappelons très brièvement quelques traits importants de son caractère et de sa vie, indépendants de la musique.

Premièrement la fuite de la Bohème antiréformiste vers Marbourg Wallerstein-Oettingen en Souabe, d'un garçon de onze ans précocement orphelin n'ayant sur lui aucun document, pour retrouver son oncle Joseph, comme il le décrit dans les Notes (p. 14 et suivantes) a été une sorte d'aventure romantique admirable. Cette décision clef caractérise manifestement son courage inné à poursuivre le but tracé même au prix de certains risques personnels, son aspiration à la liberté, une décision libre de son destin et de ses projets qu'il a manifestés de nouveau quand il s'est opposé à son oncle qui lui défendait de devenir musicien.

Ses courtes études mais qui l'influencèrent beaucoup à l'Université de Bonn, ont encore fortifié ces traits de caractère innés et ces inclinations. En lui faisant connaitre la philosophie contemporaine et classique, la littérature et les sciences naturelles, ses études universitaires ont élargi son horizon musical limité. La philosophie du siècle des lumières, rationaliste (Kant), lui a enseigné la curiosité critique, l'analyse systématique et la compréhension éthique de la vie et de l'oeuvre. Cependant le rationalisme des écoles allemandes n'a pas étouffé sa sensibilité innée (peut-être un des traits de son origine tchèque et slave) qu'il considérait comme un caractère et une condition fondamentale pour l'art et la musique nouvelle (il a exprimé sa propre conception poétique entre autre dans les considé. rations qu'il a appelées: Sur la musique comme art purement sentimental, avec des remarques philosophiques et critiques sur les opérations morales de notre être).

A. J. Reicha n'a pas été socialement du type de musicien-serviteur comme l'avait créé le XVIII e siècle en Europe centrale et comme l'était encore son oncle Joseph Reicha et plus ou moins aussi Joseph Haydn.

14 Se rapporte aussi à J. L. Dusík qui composait exclusivement de la musique profane. Dans la branche des compositeurs tchèques de la fin du XVIIIe et du commencement du XIXe siècle y compris V. J. Tomášek, la musique religieuse est représentée par de nombreuses oeuvres. 
Avec ses emplois futurs, surtout comme musicien et comme maître de musique et compositeur (sans dépendance de la classe gouvernante aristocratique), avec ses opinions d'esprit libre, et sa conception éthique de l'oeuvre, il est devenu plutôt "musicien-citoyen" dans le sens des idées de $l^{\prime}$ Epoque des lumières, des changements sociaux de la fin du XVIII e et du début du XIX $\mathrm{X}^{\mathrm{e}}$ siècle, c'est-à-dire un type de compositeur tel que l'a pu créer l'époque de la bourgeoisie.

Son séjour de six ans à Hambourg au cours duquel il s'est lié étroitement avec des artistes et des musiciens français, a été décisif pour sa formation et son statut social. Il a bientôt renoncé à l'espoir réel de devenir le useul héritier légitime" de son oncle comme musicien au service de l'Electeur de Cologne Maximilien d'Autriche (Notes, p. 16). A Hambourg il a trouvé un autre moyen de gagner sa vie, peut-être plus modeste, mais plus favorable du point de vue de la création, celui de maître de musique dans des familles de notables de la ville. Plus tard A. J. Reicha n'a jamais accepté d'être au service permanent de la société aristocratique et ne l'a même jamais recherchée. S'il a dû chercher pour des raisons sociales des liens chez la noblesse ou les familles régnantes (pendant son séjour à Vienne) il l'a alors fait en dédicaçant ses oeuvres (comme par exemple L'Art de varier ou 57 Variations sur un thème d'invention, composés et dédiées à Son Altesse Royale Monsieur le Prince Louis Ferdinand de Prusse, op. 57, 1803 - 04). Par cette attitude envers les représentants de la vieille société, A. J. Reicha ressemble tout à fait au compagnon artistique de sa jeunesse $L$. van Beethoven.

A Hambourg ses sentiments patriotiques ont commencé à se cristalliser. Ils se manifestent par son inclination pour la France, la langue et la culture française et aussi par les possibilités que semblaient offrir à l'art et à la musique les transformations radicales de l'ordre social de ce pays. A. J. Reicha a composé à Hambourg son premier opéra "français« Obaldi ou les Français en Egypte (par une bizarre coïncidence probablement une année avant l'expédition en Egypte de Bonaparte). Dans son premier cahier de fugues pour piano (1799) il écrit une préface en français pleine d'admiration et d'amour pour la France et de confiance dans sa force culturelle. Dans une fugue pour piano il prend comme thème l'air 'd'une chanson populaire française (Alsacienne). L'éducation d'A. J. Reicha dans un esprit français due d'abord à l'influence de Lucie Certelet, sa tante, la femme de Joseph Reicha, a été certainement renforcée par de puissantes impulsions. La francophilie d'A. J. Reicha n'a pas été motivée seulement par le nationalisme. Aux yeux d'A. J. Reicha la France représentait le pays dans lequel il pouvait réaliser ses idées sur la musique et sur ses fonctions culturelles. Evidemment A. J. Reicha a été influencé par les musiciens et les compositeurs français qui obtenaient des places importantes dans les institutions nées du mouvement révolutionnaire de l'époque. A ces musiciens il a aussi dédié ses nouvelles oeuvres (par exemple Douze Fugues pour piano composées et dédiées aux citoyens Méhul, Cherubini, Gossec, Le Sueur et Martini, inspecteurs du Con. servatoire à Paris, 1799 et autres). 
L'association des noms de ces musiciens du "Tiers Etat" (Gossec, Méhul, Cherubini étaient vraiment à l'époque de la composition des premières fugues d'A. Reicha des compositeurs de la Révolution et de la République) avec le recueil d'A. J. Reicha n'a pas été superficielle. A. J. Reicha a trouvé en lui même assez de force pour s'identifier au mouvement révolutionnaire en tant que musicien et surtout en tant que musi. cien. Il n'a pas choisi les mêmes voies que Gossec et ses compagnons qui ont composé par exemple pour le $1^{\mathrm{er}}$ anniversaire de la prise de la $\mathrm{Ba}-$ stille, le Chant du 14 Juillet. La pensée d'exprimer les nouvelles idées par la musique, éventuellement par la musique liée au texte ne l'a jamais quitté et elle est devenue partie intégrante de ses vues sur l'art.

Il a commencé à réaliser ce qu'il s'était proposé dans un domaine considéré comme le moins favorable: avec une fugue c'est-à-dire avec une forme uniquement instrumentale que les classiques cessaient de composer ou ne composaient qu'exceptionnellement (avant A. J. Reicha, Mozart dans la symphonie en do majeur KV 551, après A. J. Reicha, Beethoven dans les derniers quatuors). A. J. Reicha n'a pas provoqué une renaissance histortique de la fugue baroque comme l'ont fait au $\mathrm{XX}^{\mathrm{e}}$ siècle quelques néoclassiques (Hindemith, Chostakovitch) il voulait, en conservant les caractères formels de la fugue (division en 3 parties, thème unique, polyphonie) donner à ses fugues une "valeur esthétique« plus élevée, il voulait même écrire un nouveau pendant français aux fugues, telles que les avaient créées les grands maitres J. S. Bach, G. F. Haendel (dans la préface dejà citée du cahier des fugues de 1799 repris dans le traité théorique Ueber das neue Fugensystem. Als Zusatz zu dem Werke unter dem Titel: Trente-six fugues pour pianoforte, composées d'après un nouveau système 1805, il écrit: "Peut-être est-il un jour réservé à la Nation Française de donner à la Fugue un nouvel éclatı).

A. J. Reicha a écrit environ 80 fugues (la plupart pour piano). Ce nombre montre clairement quelle importance il donne à cette forme musicale et combien il l'aimait. La vraie signification de ces fugues dans l'orientation consciente d'A. J. Reicha pour les nouvelles idées artistiques est exprimée alors dans leur contenu musical qu'il avait dejà mis au point dans les premiers cahiers de fugues pour piano de 1799 et 1803. Les fugues d'A. J. Reicha sont nouvelles par leur structure non traditionnelle non seulement sur des thèmes diatoniques et chromatiques assymétriques mais aussi symétriques, sur des thèmes majeurs, mineurs et modaux avec des thèmes musicaux simples ou composés, étant donné qu'il ne tient pas compte de la règle de l'entrée tonale des voix dans l'exposition (répercussion) ni du plan sévère tonal et modal dans l'exposition, le développement, le strette. Quelques unes de ces fugues sont tonalement et modalement si mouvantes qu'il n'a pas indiqué leur mode.15 A. J. Reicha compose ses fugues dans les mesures $2 / 8,5 / 8,6 / 8$ et $3 / 4,12 / 4,4 / 2$. Toutes sont

15 No 12, 15, 31. Aucune des 36 fugues de Reicha n'est atonale comme l'a jugé J. V. Sýkora dans la discussion: Critical Years in European Music History 1800 -1820, imprimé in: Report of the Tenth Congress Ljubljana 1967, ed. D. Cvetko, p. 212. 
efficaces au point de vue artistique et enrichissent la musique de leur genre.

La valeur des fugues pour piano des années 1799 à 1803 est beaucoup plus grande et significative. Elles posent les bases d'une nouvelle poétique musicale et des principes de composition niant quelques normes fondamentales du style de l'Ecole viennoise classique. Certaines parties de l'oeuvre d'A. J. Reicha individualisent aussi son langage musical. A. J. Reicha se rend bien compte des nouveaux traits individuels de la poétique musicale de ses fugues et en parle non seulement dans l'essai Ueber das neue Fugensystem (1805), mais aussi dans d'autres oeuvres théoriques, et plus tard comme maître de musique de composition il leur assigne un rôle d'évolution important. Les fugues pour piano d'A. J. Reicha ont cependant ouvert la voie à de nouveaux principes poétiques aussi dans d'autres oeuvres.

Maurice Emmanuel, auteur français de la biographie d'A. J. Reicha a publié dans son livre Antonin Reicha (Paris 1937, p. 104-5) les manuscrits posthumes des deux premières pages de la partition de l'ouverture en ré majeur dont le thème principal d'ouverture est composé dans la mesure $5 / 8$, comme dans la fugue $\mathrm{n}^{0} 20$ du cahier de 36 fugues pour piano. Emmanuel attire aussi l'attention sur l'apparition d'autres éléments de la nouvelle poétique musicale dans d'autres oeuvres instrumentales postérieures d'A. J. Reicha. Olga šotolová, musicologue tchèque, dans son article Antonin Rejcha pedagog a skladatel - "Antoine Reicha pédagogue et compositeuru, revient de nouveau à la mesure $5 / 8$ de l'ouverture en ré majeur (elle date de 1823 la naissance de cette oeuvre) et fait remarquer (de même que l'avait fait $\mathrm{M}$. Emmanuel) un autre trait caractéristique de la poétique musicale d'A. J. Reicha, à savoir son sens et également son talent à chercher et à découvrir de nouvelles combinaisons de sons, d'effets et de groupements d'instruments. A. J. Reicha a été, sans aucun doute, un excellent instrumentaliste. Dans ses oeuvres symphoniques et de musique de chambre il ne s'est pas contenté d'un groupement d'instruments classiques fixé. La formation du quintette pour instruments à vent qu'il a stabilisée n'est qu'une des preuves du dynamisme de sons, d'instruments et d'invention dû à son talent. Dans le genre musique de chambre nous pouvons faire une remarque sur d'autres oeuvres d'A. J. Reicha dans lesquelles il se présente comme un novateur inépuisable des plus diverses combinaisons d'instruments et de groupements d'instruments qu'il emploie avec maîtrise, du duo au nonette et à la symphonie pour orchestre de chambre. Maurice Emmanuel dans une note sur un autre exemple d'une oeuvre symphonique à programme, nommée "Musique pour célébrer la mémoire des grands hommes qui se sont illustrés au service de la $\mathrm{Na}$ tion Française lequel travaille le compositeur et dénombre 60 violons, 18 violes, 18 violoncelles, 18 contrebasses, 12 flûtes, 12 hautbois, 12 clarinettes, 12 fagots, 12 cors d'harmonie, 6 trompettes, 6 trombones, 6 paires de tympans. Il a choisi cette distribution d'orchestre non seulement pour atteindre l'intensité d'effet du son, mais aussi pour les possibilités d'utilisation des com- 
binaisons les plus différentes de timbre des instruments pris individuellement ou par groupes.

"L'Ode symphonique» à la mémoire et à la gloire du peuple français et des serviteurs émérites de la Nation illustre d'autres traits importants de la poétique musicale de A. J. Reicha et son orientation vers la musique romantique du type à programme. A. J. Reicha a participé aussi à une grande oeuvre commencée par Beethoven laquelle avec ses symphonies à programme a enrichi l'élève d'A. J. Reicha, Hector Berlioz. Cette oeuvre commune n'a pas eu son importance seulement pour la naissance de la musique à programme, mais elle a en général enrichi les possibilités d'expression de la musique. Grâce aux efforts de Beethoven, de Reicha (et d'autres musiciens des débuts du romantisme) de Berlioz (le premier géant de la génération des vrais romantiques) la musique a pu devenir un art qui recherchait un nouveau rapport avec la poésie, le théâtre et la philosophie. Dans les genres synthétiques, vocal et dramatique, dans ia liaison organique avec le contenu du texte, la musique, il est vrai, perdait cette indépendance qu'elle avait encore dans les opéras de Mozart, dans les oratorios de Haydn mais d'autre part en se rapprochant de la poésie, du théâtre et de la philosophie elle devenait un art expressif.

Pour A. J. Reicha la littérature, le théâtre et la philosophie étaient une réalité proche. De nombreuses oeuvres théoriques témoignent de ses dons littéraires, ainsi que l'ode dédicatoire française à Joseph Haydn écrite en hommage à son maître viennois et qu'il a placée en tête de l'édition viennoise de ses fugues pour piano. Pour l'oeuvre vocale et dramatique d'A. J. Reicha, ses considérations sur la déclamation musicale surtout française ont une importance fondamentale. Si dans beaucoup d'oeuvres d'A. J. Reicha nous trouvons de nombreuses pièces vocales et dramatiques ( 9 opéras), il ne faut voir dans ce fait que la réalisation d'un des nombreux devoirs que posaient au compositeur le développement artistique et le goût du public bourgeois à la limite du XVIIIe et du XIX ${ }^{\mathrm{e}}$ siècle.

A. J. Reicha a écrit les airs concertants italiens dans le vieux style classique. Son opéra italien Argina, regina di Grenata sur le livret de Metastasio, composé à la demande de la cour impériale a sans aucun doute suivi comme ligne directive celle de l'opéra seria du XVIIIo siècle. Dans son autobiographie A. J. Reicha reconnaît qu'il a été victime, comme compositeur d'opéras, des intrigues commerciales des théâtres parisiens et qu'il a dû accepter que son opéra seria Cagliostro ou les Illuminés soit transformé en opéra bouffe (aussi avec la contribution de la musique d'un autre auteur V. Ch. P. Dourlen). A. J. Reicha a sans aucun doute payé sa dette aux manières et au goût de l'empire napoléonien, mais personnellement et artistiquement ces obligations ne lui convenaient pas. Son idéal n'a jamais été "un opéra dont le seul but est de divertir le public pendant quelque temps, n'est autre chose qu'une marchandise de mode» (Notes, p. 32). A. J. Reicha a cherché théoriquement (déjà dans le traité Philosophisch-praktische Anmerkungen zu den praktischen Beispielen, 1803) et aussi pratiquement une nouvelle esthétique de l'opéra. Le principe fondamental de cette esthétique devait être l'harmonisation et la subordina- 
tion de la musique aux exigences de la vérité dramatique. A. J. Reicha a aussi examiné et approfondi les principes de la déclamation française. ${ }^{16}$

Les opéras Nathalie ou la famille russe (1816), Sapho (1822), et Philoctète (1819) sont écrits dans le style du grand opéra, et ils représentent la réalisation artistique des idées d'A. J. Reicha dans le domaine et l'esthétique de l'opéra. Aujourd'hui, ne connaissant pas leur réalisation, nous ne pouvons qu'estimer que la progressivité et la nouveauté des idéaux artistiques d'A. J. Reicha dans l'évolution et la naissance de l'opéra romantique, surtout français, ne sont pas négligeables. En jugeant d'après quelques impressions que des scènes de l'opéra Sapho ont laissées dans la mémoire de Berlioz ${ }^{17}$ nous pourrions affirmer que les oeuvres d'opéra dominantes de Reicha appartiennent non seulement à l'histoire de l'opéra comme partie importante de son évolution, mais en même temps donnent une autre preuve de l'art exceptionnel de son auteur.

Nos considérations sur Antoine Joseph Reicha, l'ami de Beethoven et son contemporain, avaient pour but de réhabiliter A. J. Reicha comme compositeur. Nous ne nous sommes permis de faire la comparaison avec L. van Beethoven que pour des raisons biographiques extérieures car ces deux compositeurs appartiennent l'un comme l'autre, aussi bien par les moyens stylistiques de leur oeuvre que par les rapports dynamiques et peut-être révolutionnaires, à l'école classique viennoise. Dans cette voie Beethoven est allé plus en avant et il a créé de grandes oeuvres symphoniques, de chambre et concertantes. De même le seul opéra de Beethoven Fidelio, bien qu'il n'ait pas résolu l'esthétique de l'opéra post-classique, témoigne du génie unique de son auteur. A. J. Reicha auteur de fugues pour piano, de quintettes pour instruments à vent, de pièces pour ensernbles de chambre les plus différents, de symphonies et d'opéras n'a jamais atteint le génie de Beethoven. Il n'a pas été cependant qu'un simple satellite artistique de son ami de Bonn, de son compagnon viennois, et son soutien, comme on le juge encore quelquefois dans la littérature lexicographique et historiographique.

\section{POVZETEK}

V zgodovinopisju in leksikalni literaturi je skladatelj češkega rodu A. J. Reicha (1770-1835) na splošno obravnavan kot teoretik in pedagog. Glede na to, da je ta vsestranski glasbenik napisal vrsto del $s$ področja teorije glasbe in na pariškem konservatoriju vzgojil tako znamenite skladatelje kot so bili npr. $H$. Berlioz, C. Franck, Ch. Gounod in drugi, je to tudi prav. Reicha je umetniško

16 Pour cela comparer Alena Krutová: Opera $v$ pojetí Antonína Rejchy "L'opéra dans la conception d'Antoine Reicha", Brno 1971 - en manuscrit, ou sont étudiés les problèmes de la déclamation musicale aussi bien dans ses opéras que dans son oeuvre théorique Art du compositeur dramatique.

17 Le romantique Berlioz dans ses Mémoires (1870) et peu de temps auparavant dans la Gazette Musicale a comparé Sapho avec les quintettes pour instruments à vent "classiques" qu'il considère comme oeuvres wintéressantes mais un peu froidesu tandis qu'il considère le duo de Sapho comme "magnifique" aussi bien que les choeurs (»je me souviens d'y avoir admiré plusieurs choeurs d'une belle couleur antique et un duo d'une rare énergie»), comparer Maurice Emmanuel, oeuvre citée, p. 55 . 
rasel $\mathrm{v}$ Bonnu in na Dunaju, študiral pa $\mathrm{z} \mathrm{L}$. v. Beethovnom, $\mathrm{s}$ katerim se je tudi spoprijateljil. Beethoven je bil ta, ki je Reichove skladbe priporočil svojemu založniku Breitkopfu in Härtlu $v$ Leipzigu. Razni zunanji in notranji razlogi so vplivali, da je Reicha kot skladatelj stal $v$ senci velike Beethovnove umetnosti. Reicho je glasbenemu svetu pravzaprav posredovala beethovenska literatura, ki ga je seveda interpretirala kot satelitski pojav dunajske klasične šole.

Reichovo privrženost tej šoli izpričuje znaten del njegovih instrumentalnih kompozicij, posebno sonate, kvarteti, kvinteti, uverture, simfonije in drugo. V le-teh avtor nikjer ni šel prek norm Haydnovega in Mozartovega klasicizma in tudi ne zgodnjega Beethovna. Kot skladatelj 25 pihalnih kvintetov pa si je pridobil edinstveno mesto. V nekaterih svojih poznejših simfonijah in uverturah se kažejo zanimivi stilni prijemi, s katerimi se Reicha oddaljuje od stilnega okvira dunajskih mojstrov. To predvsem kar se tiče kromatike in modalnosti melodije in harmonije, ritmične in taktovne wasimetrije» in neklasicističnega pojmovanja glasbene forme. Korene te posebne Reichove stilne orientacije moremo iskati že v nekaterih skladbah hamburškega razdobja (1794-1799), zlasti v klavirskih fugah. Hotel je namreč oblikovati "francoske» fuge, take, ki bi se po slogu in duhu razlikovale od strogih Bachovih in Händlovih. V Hamburgu je tudi nastala prva Reichova francoska opera, prva $v$ vrsti njegovih vokalnih in dramatičnih skladb na francoski tekst, $\mathbf{s}$ katerimi je hotel prispevati $\mathrm{k}$ nastajanju francoske glasbe njene zgodnje romantične faze. Ko se je 1809 ustalil v Parizu in se v vsem vključil $\mathrm{v}$ tamošnje kulturno vzdušje, je tu našel razumevanje s strani francoskih interpretov in učencev. Berlioz je na primer navdušeno ocenil njegove zadnje opere (posebno Sapho). Vendar je za Fétisa in še mnoge druge bil eksponent dunajske (oz. nemške) šole in zato ni bil kdove kako sprejemljiv. Ni ga razumelo niti pariško operno občinstvo, zato ne, ker se je trudil za nekonvencionalno pojmovanje opernega žanra.

Reicha ni nikoli dosegel veličine svojega bonnskega in dunajskega prijatelja Beethovna. Pomen, ki mu gre kot skladatelju, pa je zmanjšala ravno primerjava $s$ tem mojstrom. Po tej strani ni bil nič manjši kot še vrsta manj pomembnih skladateljev, $\mathrm{ki}$ tudi štejejo $\mathrm{v}$ dunajsko klasično šolo, $\mathrm{v}$ primerjavi $\mathrm{z}$ njimi je bil celo pomembnejši. Ustvaril je dela, ki še živijo in z njimi on (pihalne kvintete, fuge, simfonije). V številnem opusu, ki ga je zapustil, bi lahko mnoge skladbe smatrali kot nekaj, kar najavlja nekako novo renesanso. Popolni seznam del tega skladatelja in njegovo avtobiografijo je mogoče najti $\mathrm{v}$ ediciji J. Vysloužila, Notes sur Antoine Reicha, Brno 1970. 